\title{
VALUES OF BINARY QUADRATIC FORMS AT INTEGER POINTS AND SCHMIDT GAMES
}

\author{
DMITRY KLEINBOCK AND BARAK WEISS
}

\begin{abstract}
We prove that for any countable set $A$ of real numbers, the set of binary indefinite quadratic forms $Q$ such that the closure of $Q\left(\mathbb{Z}^{2}\right)$ is disjoint from $A$ has full Hausdorff dimension.
\end{abstract}

Dedicated to S.G. Dani on the occasion of his 65th birthday

\section{INTRODUCTION}

We start with the following statement, conjectured by Oppenheim and Davenport in the 1930s and proved by Margulis in the 1980s:

Theorem 1.1. Let $Q$ be a real nondegenerate indefinite quadratic form of $n>2$ variables which is not proportional to a rational form. Then

$$
0 \text { belongs to the closure of } Q\left(\mathbb{Z}^{n} \backslash\{0\}\right) \text {. }
$$

Margulis used an approach which was suggested earlier by Raghunathan and implicitly used earlier by Cassels and Swinnerton-Dyer CaSD. Let $Q_{0}$ be a fixed quadratic form of the same signature as $Q$, then one can write $Q(\mathbf{v})=a Q_{0}(g \mathbf{v})$ for some $g \in G=\mathrm{SL}_{n}(\mathbb{R})$ and $a \in \mathbb{R}$. Let $F$ be the stabilizer of $Q_{0}$ and let $\Gamma=\mathrm{SL}_{n}(\mathbb{Z})$. Then (1.1) holds if and only if the orbit $F g \mathbb{Z}^{n}$ in the space $G / \Gamma$ of unimodular lattices in $\mathbb{R}^{n}$ is unbounded. The theorem proved by Margulis (in the case $n=3$, to which the general case can be reduced) stated that any bounded orbit must be compact, from which it is not hard to derive Theorem 1.1.

Note that it was later proved by Dani and Margulis that for $Q$ as above the set $Q\left(\mathbb{Z}^{n}\right)$, and even $Q\left(P\left(\mathbb{Z}^{n}\right)\right.$ ), is dense in $\mathbb{R}$ (here $P\left(\mathbb{Z}^{n}\right)$ stands for the set of primitive integer points in $\left.\mathbb{Z}^{n}\right)$.

When $n=2$ it is well known that conclusion of Theorem 1.1 fails. Namely, take

$$
Q(p, q)=p^{2}-\lambda^{2} q^{2}=q^{2}\left(\frac{p}{q}-\lambda\right)\left(\frac{p}{q}+\lambda\right)
$$

such that $\lambda$ is badly approximable, that is,

$$
\inf _{p \in \mathbb{Z}, q \in \mathbb{N}} q^{2}\left|\frac{p}{q}-\lambda\right|>0
$$

then the absolute value of $Q(p, q)$ is uniformly bounded away from 0 for any nonzero integer $(p, q)$. It is known from the work of Jarník [J] that the set of such $\lambda$ 's, although null, is thick, that is, its intersection with any nonempty open set has full Hausdorff dimension. As in the reduction of the Oppenheim conjecture to a dynamical statement, choose $Q_{0}(x, y) \stackrel{\text { def }}{=} x y$, let

$$
G \stackrel{\text { def }}{=} \mathrm{SL}_{2}(\mathbb{R}),
$$

and let $F$ be the connected component of the identity in the stabilizer of $Q_{0}$, namely

$$
F=\left\{g_{t}: t \in \mathbb{R}\right\} \text { where } g_{t}=\left(\begin{array}{cc}
e^{t} & 0 \\
0 & e^{-t}
\end{array}\right) .
$$

We also let

$$
X \stackrel{\text { def }}{=} G / \Gamma \text { where } \Gamma \stackrel{\text { def }}{=} \mathrm{SL}_{2}(\mathbb{Z}), \text { and } \mathcal{Q} \stackrel{\text { def }}{=} F \backslash G \text {; }
$$

\footnotetext{
Date: September 19, 2013.
} 
thus $X$ can be identified with the space of unimodular lattices in $\mathbb{R}^{2}$, and $\mathcal{Q}$ can be identified with the space of binary indefinite quadratic forms, considered up to scaling. The set of integer values of a quadratic form is a (set-valued) function on $F \backslash G / \Gamma$ but since this double coset space has a complicated topological structure, it is more useful to consider it either as an $F$-invariant function on $X$, or dually, as a $\Gamma$-invariant function on $\mathcal{Q}$. This duality principle (already evident in the work of Cassels and Swinnerton-Dyer CaSD] and developed explicitly by Dani [D1] in a related context) makes it possible to recast dynamical properties of the $F$-action on $X$ as properties of quadratic forms. In particular (see e.g. [Ma2, Lemma 2.2.1]), it follows from Mahler's Compactness Criterion that for a quadratic form $Q(\mathbf{v})=Q_{0}(g \mathbf{v}) \in \mathcal{Q}$, the set of values $Q\left(\mathbb{Z}^{2}\right)$ has a gap at zero if and only if the orbit $F\left(g \mathbb{Z}^{2}\right)$ is bounded in $X$. In light of this Jarník's theorem implies:

Theorem 1.2. Let $X$ and $Q_{0}$ be as above. Then the set

$$
\left\{x \in X: 0 \notin \overline{Q_{0}(x \backslash\{0\})}\right\}
$$

is thick; dually, the set of binary indefinite quadratic forms whose set of values at integer points has a gap at 0 is thick in the space of all binary indefinite forms.

It is worth pointing out that a generalization of this argument is due to S.G. Dani D1, D2, who used W. Schmidt's results and methods S1, S2 to find thick sets of points with bounded orbits in other homogeneous spaces. See also [Ar, $\mathrm{KM}$, KW2 for further developments.

The goal of this paper is to strengthen Theorem 1.2 by considering even more complicated properties of the sets of values of quadratic forms at integer points, and, correspondingly, the behavior of $F$-orbits in $X$. Here is one of our main results:

Theorem 1.3. For any countable subset $A \subset \mathbb{R}$, the set

$$
\left\{x \in X: \overline{Q_{0}(x \backslash\{0\})} \cap A=\varnothing\right\}
$$

is thick. Consequently, the set of binary indefinite quadratic forms whose set of values at integer points miss a given countable set is thick in the space of all binary indefinite forms.

Note that by a theorem of Lekkerkerker $[\mathrm{L}$, for $Q$ as in (1.2) the set of accumulation points of $Q\left(\mathbb{Z}^{2}\right)$ is discrete if and only if $\lambda$ is quadratic irrational. See [TV], DN] for a precise description of the sets of accumulation points in these cases.

We will derive Theorem 1.3 from its more general dynamical counterpart. To state it, we need the following definition. Let $H$ be a connected subgroup of $G$ different from $F$, and let $Z$ be a submanifold of $X$. Say that $Z$ is $(F, H)$-transversal if the following two conditions hold:

$(F)$ for any $x \in Z, T_{x}(F x)$ is not contained in $T_{x} Z$;

$(H, F)$ for any $x \in Z, T_{x}(H x)$ is not contained in $T_{x} Z \oplus T_{x}(F x)$.

For example, the above conditions are satisfied if $Z$ consists of a single point. If $\operatorname{dim}(Z)=$ 1 , the $(F, H)$-transversality of $Z$ is equivalent to saying that for each $x \in Z$ the lines $T_{x}(H x)$, $T_{x} Z$ and $T_{x}(F x)$ are in general position, i.e. they generate the space $T_{x} X$.

We permit $Z$ to be a manifold with boundary; in such a case, smoothness of maps and definitions of tangent spaces at the boundary points are defined by positing the existence of smooth extensions (see e.g. GP, Chap. 2]). We also note that in the application to quadratic forms, $Z$ is an analytic manifold, but in all our arguments, it suffices to assume that $Z$ is $C^{1}$-smooth.

Now let us denote by $\mathrm{H}^{+}$and $H^{-}$the expanding and contracting horospherical subgroups with respect to $g_{1}$ :

$$
H^{+} \stackrel{\text { def }}{=}\left\{h_{s} \stackrel{\text { def }}{=}\left(\begin{array}{ll}
1 & s \\
0 & 1
\end{array}\right): s \in \mathbb{R}\right\}, \quad H^{-\stackrel{\text { def }}{=}}\left\{\left(\begin{array}{ll}
1 & 0 \\
s & 1
\end{array}\right): s \in \mathbb{R}\right\} .
$$

A dynamical statement which will imply Theorem 1.3 is as follows: 
Theorem 1.4. Let $Z$ be a countable union of submanifolds of $X$ which are both $\left(F, H^{+}\right)$and $\left(F, H^{-}\right)$-transversal. Then the set

$$
\{x \in X: F x \text { is bounded and } \overline{F x} \cap Z=\varnothing\}
$$

is thick.

Note that for arbitrary homogeneous spaces $X=G / \Gamma$, non-quasiunipotent subgroups $F$ and finite sets $Z$ the thickness of the set (1.7) was conjectured by Margulis Ma1. Then in [KM it was shown that, for mixing flows, the set of points with bounded orbits is thick, and in $\left[\mathrm{K}\right.$, for arbitrary homogeneous spaces and $Z$ being a finite union of $Z_{i}$ as in the above theorem 1 - that the set

$$
\{x \in X: \overline{F x} \cap Z=\varnothing\}
$$

is thick. Margulis' Conjecture was finally proved in KW2 using the technique of Schmidt games, with the set $Z$ upgraded from finite to countable. However the argument of KW2 could not produce a result for $Z$ being more than zero-dimensional, which, in particular, is needed for an application to quadratic forms.

Organization of the paper. In $\$ 2$ we explain the reduction of Theorem 1.3 to Theorem 1.4. In $\$ 3$ we describe a variant of Schmidt's $(\alpha, \beta)$-game. This variant is very close to the absolute game and hyperplane absolute game introduced in [Mc and BFKRW] respectively, but adapted to a situation in which we want to play on a smooth manifold. In $\$ 4$ we state our main technical result, Theorem 4.3 which implies Theorem 1.4 and is a result on the winning property of a certain set for the above game. We complete the proof of Theorem 4.3 in $\$ 5$.

Acknowledgements. The authors gratefully acknowledge the support of BSF grant 2010428, ERC starter grant DLGAPS 279893, and NSF grant DMS-1101320.

\section{Dynamics and gaps Between Values of Quadratic Forms}

In this section we explain how to reduce Theorem 1.3 to Theorem 1.4 As was mentioned in the introduction, the equivalence between the set of values of the form being bounded away from 0 and the $F$-orbit of the corresponding lattice being bounded in $X$ is well-known. We need to understand how to dynamically describe the set of quadratic forms whose values miss a fixed $a \neq 0$. So fix a nonzero $a$ and choose $\mathbf{v} \in \mathbb{R}^{2}$ such that $Q_{0}(\mathbf{v})=a$. Not much will depend on this choice, yet the most obvious one seems to be choosing

$$
\mathbf{v}=\left\{\begin{array}{l}
(\sqrt{a}, \sqrt{a}) \quad \text { if } a>0 \\
(-\sqrt{a}, \sqrt{a}) \text { otherwise }
\end{array}\right.
$$

which is what we will do. Now define

$$
\tilde{Z}_{\mathbf{v}} \stackrel{\text { def }}{=}\{x \in X: \mathbf{v} \in x\},
$$

that is, the set of unimodular lattices in $\mathbb{R}^{2}$ containing $\mathbf{v}$. The structure of this set is easy to describe: $\tilde{Z}_{\mathbf{v}}=\bigcup_{n \in \mathbb{N}} Z_{\frac{1}{n} \mathbf{v}}$ where

$$
Z_{\mathbf{v}} \stackrel{\text { def }}{=}\{x \in X: \mathbf{v} \in P(x)\},
$$

the set of unimodular lattices in $\mathbb{R}^{2}$ containing $\mathbf{v}$ as a primitive vector. The latter is simply a closed horocycle, namely a periodic orbit of the subgroup $V$ of $G$ stabilizing $\mathbf{v}$, which is

\footnotetext{
${ }^{1}$ Technically the statement in $\left[\mathrm{K}\right.$, Corollary 4.4.2] is weaker than quoted here, namely $Z_{i}$ are assumed to satisfy condition $(F)$ and have dimension smaller than dimensions of $\mathrm{H}^{+}$and $\mathrm{H}^{-}-$but the argument relies precisely on the combination of $\left(F, H^{+}\right)$- and $\left(F, H^{-}\right)$-transversality.
} 
easily seen to be, for our choice of $\mathbf{v}$, equal to

$$
V \stackrel{\text { def }}{=}\left\{\begin{array}{l}
\left\{\left(\begin{array}{cc}
1+s & -s \\
s & 1-s
\end{array}\right): s \in \mathbb{R}\right\} \quad \text { if } a>0 \\
\left\{\left(\begin{array}{cc}
-1-s & s \\
s & -1+s
\end{array}\right): s \in \mathbb{R}\right\} \text { otherwise }
\end{array}\right.
$$

Now let us state a proposition which relates escaping $\tilde{Z}_{\mathbf{v}}$ with a gap in values of quadratic forms at $a$ :

Proposition 2.1. Suppose $x \in X$ is such that $F x$ is bounded, let $a \neq 0$, and define $\mathbf{v}$ by (2.1). Then $\overline{F x} \cap \tilde{Z}_{\mathbf{v}}=\varnothing$ if and only if $a \notin \overline{Q_{0}(x \backslash\{0\})}$.

Proof. For the 'if' direction, suppose that $x_{0} \in \overline{F x}$ contains v. Then there are $t_{n}$ such that $g_{t_{n}} x \rightarrow x_{0}$ and so there are $\mathbf{w}_{n} \in x$ with $g_{t_{n}} \mathbf{w}_{n} \rightarrow \mathbf{v}$. In particular $\mathbf{w}_{n} \neq 0$. Hence $Q_{0}\left(\mathbf{w}_{n}\right)=Q_{0}\left(g_{t_{n}} \mathbf{w}_{n}\right) \rightarrow Q_{0}(\mathbf{v})=a$, so $a \in \overline{Q_{0}(x \backslash\{0\})}$, a contradiction.

Suppose the converse does not hold, that is, there exist vectors $\mathbf{v}_{k} \in x$ such that $Q_{0}\left(\mathbf{v}_{k}\right) \rightarrow$ $a$ as $k \rightarrow \infty$. For each $k$, choose $t_{k} \in \mathbb{R}$ such that $g_{t_{k}} \mathbf{v}_{k}$ belongs to the line passing through $\mathbf{v}$; then it is easy to see that

$$
g_{t_{k}} \mathbf{v}_{k} \rightarrow \mathbf{v} \text { as } k \rightarrow \infty .
$$

But $F x$ is relatively compact, hence there exists a limit point $x_{0}$ of the sequence $g_{t_{k}} x$ of lattices, and from (2.3) it follows that $x_{0}$ contains $\mathbf{v}$, or, equivalently, $x_{0} \in \tilde{Z}_{\mathbf{v}}$, contrary to assumption.

Remark 2.2. The boundedness of $F x$ was only used in proving the implication $\Longrightarrow$. If $F x$ is not bounded (or equivalently, $0 \in \overline{Q_{0}(x \backslash\{0\})}$ ) then the situation is more interesting. A simple argument involving multiplication by integers, shows that when $Q_{0}(x \backslash\{0\})$ contains sequences approaching 0 from both sides, then the set of values $Q_{0}(x \backslash\{0\})$ is dense. However, as shown by Oppenheim [O], lattices $x$ for which 0 is only a one-sided limit of $Q_{0}(x \backslash\{0\})$ do exist.

We record the following:

Lemma 2.3. For $\mathbf{v}$ as in (2.1) (in fact, the same is true for every $\mathbf{v}$ with $\left.Q_{0}(\mathbf{v}) \neq 0\right)$, the manifold $Z_{\mathbf{v}}$ is both $\left(F, H^{+}\right)$-transversal and $\left(F, H^{-}\right)$-transversal.

Proof. It suffices to show that the Lie algebras of $F, V$ and $H^{+}$, as well as those of $F, V$ and $H^{+}$, span the Lie algebra of $G$. This is an easy computation using (1.4), (1.6) and (2.2).

We can now see that Theorem 1.3 follows from Theorem 1.4 .

Proof of Theorem 1.3 assuming Theorem 1.4. We have already mentioned that the boundedness of the $F$-orbit of $x$ implies that 0 is not in the closure of the set $Q_{0}(x \backslash\{0\})$. Now for each $a \in A \backslash 0$ take $\mathbf{v}=\mathbf{v}(a)$ as in (2.1), then, in view of Lemma 2.3. the set

$$
Z=\bigcup_{a \in A \backslash\{0\}} \tilde{Z}_{\mathbf{v}(a)}
$$

satisfies the assumption of Theorem 1.4. hence the set (1.7) is thick. On the other hand, Proposition 2.1 implies that for every $x$ from the set, $\overline{Q_{0}(x \backslash\{0\})} \cap A=\varnothing$.

\section{SCHMidT GAMES}

We first recall Schmidt's $(\alpha, \beta)$-game, introduced in [S1]. The game is played by two players Alice and Bob on a complete metric space 2 equipped with a target set $S$ and two

\footnotetext{
${ }^{2}$ In this section, following tradition, we denote this metric space by $X$; elsewhere $X$ continues to denote the space of two-dimensional unimodular lattices.
} 
fixed parameters $\alpha, \beta \in(0,1)$. Bob begins the $(\alpha, \beta)$-game by choosing $x_{1} \in X, r_{1}>0$, thus specifying the closed ball $B_{1} \stackrel{\text { def }}{=} \bar{B}\left(x_{1}, r_{1}\right)$, where

$$
\bar{B}(z, \rho) \stackrel{\text { def }}{=}\{x \in X: \operatorname{dist}(x, z) \leq \rho\} .
$$

Then Alice and Bob continue by alternately choosing $x_{i}^{\prime}, x_{i+1}$ so that

$$
\operatorname{dist}\left(x_{i}, x_{i}^{\prime}\right) \leq(1-\alpha) r_{i}, \quad \operatorname{dist}\left(x_{i+1}^{\prime}, x_{i}\right) \leq(1-\beta) r_{i}^{\prime}, \quad \text { where } \quad r_{i}^{\prime} \stackrel{\text { def }}{=} \alpha r_{i}, \quad r_{i+1} \stackrel{\text { def }}{=} \beta r_{i}^{\prime} .
$$

This implies that the closed balls

$$
A_{i} \stackrel{\text { def }}{=} \bar{B}\left(x_{i}^{\prime}, r_{i}^{\prime}\right), \quad B_{i+1} \stackrel{\text { def }}{=} \bar{B}\left(x_{i+1}, r_{i+1}\right)
$$

are nested, i.e.

$$
B_{1} \supset A_{1} \supset B_{2} \supset \cdots
$$

The set $S$ is said to be $\alpha$-winning if for any $\beta>0$ Alice has a strategy in the $(\alpha, \beta)$-game guaranteeing that the unique point of intersection $\bigcap_{i=1}^{\infty} B_{i}=\bigcap_{i=1}^{\infty} A_{i}$ of all the balls belongs to $S$, regardless of the way Bob chooses to play. It is called winning if it is $\alpha$-winning for some $\alpha$.

In BFKRW, inspired by ideas of McMullen $\underline{\mathrm{Mc}}$, the absolute hyperplane game was introduced. This modification is played on $\mathbb{R}^{d}$. Let $S \subset \mathbb{R}^{d}$ be a target set and let $\beta \in\left(0, \frac{1}{3}\right)$. As before Bob begins by choosing a closed ball $B_{1}$ of radius $r_{1}$ and then Alice and Bob alternate moves. The sets $B_{i}$ chosen by Bob are closed balls of radii $r_{i}$ satisfying

$$
r_{i+1} \geq \beta r_{i}
$$

The sets $A_{i}$ chosen by Alice are $r_{i}^{\prime}$-neighborhoods of affine hyperplanes, where the $r_{i}^{\prime}$ satisfy $r_{i}^{\prime} \leq \beta r_{i}$. Additionally Bob's choices must satisfy

$$
B_{i+1} \subset B_{i} \backslash A_{i} .
$$

Then $S \subset \mathbb{R}^{d}$ is said to be $\beta$-HAW (where HAW is an acronym for hyperplane absolute winning) if Alice has a strategy which leads to

$$
\bigcap_{i=1}^{\infty} B_{i} \cap S \neq \varnothing
$$

regardless of how Bob chooses to play; $S$ is said to be $H A W$ if it is $\beta$-HAW for all $0<\beta<\frac{1}{3}$. It is easy to see that $\beta$-HAW implies $\beta^{\prime}$-HAW whenever $\beta \leq \beta^{\prime}<1 / 3$; thus a set is HAW iff it is $\beta$-HAW for arbitrary small positive $\beta$. In the case $d=1$ hyperplanes are points, and thus the HAW property coincides with the absolute winning property ${ }^{3}$ introduced by McMullen in $\mathrm{Mc}$.

The following proposition summarizes properties of winning and HAW subsets of $\mathbb{R}^{d}$ :

Proposition 3.1. (a) Winning sets are thick.

(b) HAW implies winning.

(c) The countable intersection of $\alpha$-winning (resp., HAW) sets is again $\alpha$-winning (resp., $H A W)$.

(d) The image of a HAW set under a $C^{1}$ diffeomorphism $\mathbb{R}^{d} \rightarrow \mathbb{R}^{d}$ is HAW.

For the proofs, see [S1, Mc, BFKRW].

Remark 3.2. Note that in the hyperplane absolute version the radii of balls do not have to tend to zero, therefore $\cap_{i} B_{i}$ does not have to be a single point. However the outcome with radii not tending to 0 is clearly winning for Alice as long as $S$ is dense. Thus in all the proofs of the HAW property it will be safe to assume that Bob plays so that $r_{n} \rightarrow 0$ as $n \rightarrow \infty$ : indeed, if Alice has a strategy which is guaranteed to win the game whenever $r_{n} \rightarrow 0$, then the target set must be dense, and hence the strategy is guaranteed to work even if $r_{n} \nrightarrow \rightarrow 0$.

\footnotetext{
${ }^{3}$ More generally, the paper BFKRW] introduced $k$-dimensional absolute winning for every $0 \leq k<d$; the case $k=0$ was considered earlier by McMullen.
} 
We will be interested in playing variants of the two games described above on a differentiable manifold. Note that a manifold is not equipped with an intrinsic metric, nor with an intrinsic notion of affine submanifolds, and thus the definitions given above cannot be applied directly. Our approach will be to work in a given coordinate system and argue using Proposition 3.1 that the class of winning sets does not depend on the choice of a coordinate system. It will be technically simpler to work with the hyperplane absolute game and this is all that we require for the present paper. We proceed to the details.

We first define the absolute hyperplane game on an open subset $W \subset \mathbb{R}^{d}$. This is defined just as the absolute hyperplane game on $\mathbb{R}^{d}$, except for requiring that Bob's first move $B_{1}$ be contained in $W$. If Alice has a winning strategy, we say that $S$ is $H A W$ on $W$. Now suppose $X$ is a $C^{1} d$-dimensional manifold equipped with an atlas of charts $\mathcal{A}=\left(U_{\alpha}, \varphi_{\alpha}\right)$; that is, $X$ is a separable topological space, the sets $U_{\alpha}$ are open subsets of $X$ with $X=\bigcup_{\alpha} U_{\alpha}$, each $\varphi_{\alpha}: U_{\alpha} \rightarrow \mathbb{R}^{d}$ is a homeomorphism onto its image, and the transition functions $\varphi_{\beta} \circ \varphi_{\alpha}^{-1}: \varphi_{\alpha}\left(U_{\alpha} \cap U_{\beta}\right) \rightarrow \mathbb{R}^{d}$ are $C^{1}$. To define the absolute hyperplane game on $(X, \mathcal{A})$, we specify a target set $S \subset X$. Bob begins play by choosing one coordinate chart $(U, \varphi)=\left(U_{\alpha}, \varphi_{\alpha}\right)$ in $\mathcal{A}$ and a closed ball $B_{1} \subset \mathbb{R}^{d}$ contained in $\varphi(U)$. From this point on the game continues as before, where Alice and Bob alternate moves in $\varphi(U) \subset \mathbb{R}^{d}$. To decide the winner they check whether the point of intersection $\bigcap_{i} B_{i}$ belongs to $\varphi(S)$. If Alice has a winning strategy, we say that $S$ is $H A W$ on $(X, \mathcal{A})$.

Note that when $W$ is an open subset of $\mathbb{R}^{d}$, the definition of the game on $W$, given at the beginning of the previous paragraph, coincides with the definition of the game on $(X, \mathcal{A})$ if we take $X=W$ and take $\mathcal{A}$ to be the atlas consisting of one chart $(W, \mathrm{Id})$. Also note that Bob has been given the additional prerogative of choosing a coordinate chart within which to work, at the start of play, and this appears to make the winning property very restrictive. However we have:

Proposition 3.3. Suppose $X$ is a $C^{1}$ manifold with an atlas $\mathcal{A}$ and $\left(U_{i}, \varphi_{i}\right)$ is a system of coordinate charts in $\mathcal{A}$, such that $X=\bigcup U_{i}$, and $S \subset X$. Then $S$ is $H A W$ on $(X, \mathcal{A})$ if and only if for each $i, \varphi_{i}(S)$ is $H A W$ on $\varphi_{i}\left(U_{i}\right)$.

Proof. The direction $\Longrightarrow$ is immediate from the definitions, since Bob may select to work with each of the charts $\varphi_{i}$ on his first move. For the direction $\Longleftarrow$, note first that by Proposition 3.1 (a,b), $\varphi_{i}(S)$ is dense in each $\varphi_{i}\left(U_{i}\right)$ and hence $S$ is dense in $X$. Now suppose Bob chose to work in some chart $\varphi_{\alpha}$ distinct from the $\varphi_{i}$. If the diameters of the balls $B_{n}$ chosen by Bob do not decrease to zero, then $\bigcap B_{n}$ has interior. Since $S$ is dense in $X$, each $\varphi_{\alpha}(S)$ is dense in $\varphi_{\alpha}\left(U_{\alpha}\right)$, so Alice wins. Otherwise, since $B_{1}$ is compact and is covered by the open sets $\varphi_{\alpha}\left(U_{i}\right)$, there is a Lebesgue number for this cover, that is $\delta>0$ such that each subset of $B_{1}$ of diameter at most $\delta$ is contained in one of the $\varphi_{\alpha}\left(U_{i}\right)$. Thus there are $n, i$ such that

$$
\varphi_{\alpha}^{-1}\left(B_{n}\right) \subset U_{i}
$$

In light of Proposition 3.1(d), $\varphi_{\alpha}(S)=\varphi_{\alpha} \circ \varphi_{i}^{-1}\left(\varphi_{i}(S)\right)$ is HAW on $\varphi_{\alpha}\left(U_{\alpha} \cap U_{i}\right)$. In light of (3.2), the latter set contains Bob's choice $B_{n}$ and so (applying her strategy for the case that $B_{n}$ is the first ball chosen by Bob), Alice wins in this case as well.

Note that our definition above depended on the choice of atlas $\mathcal{A}$. We now deduce from Proposition 3.3 that the HAW property in fact depends only on the manifold structure on $X$, and not on a specific atlas. Namely, recall that two atlases of charts $\mathcal{A}_{1} \stackrel{\text { def }}{=}\left(U_{\alpha}, \varphi_{\alpha}\right)$, $\mathcal{A}_{2} \stackrel{\text { def }}{=}\left(V_{\beta}, \psi_{\beta}\right)$ on the same manifold $X$ are said to be $C^{1}$-compatible if $\mathcal{A}_{1} \cup \mathcal{A}_{2}$ is also a $C^{1}$-atlas of in the above sense.

Corollary 3.4. Suppose $\mathcal{A}_{1}, \mathcal{A}_{2}$ are two compatible atlases of charts, and $S \subset X$. Then $S$ is $H A W$ on $\left(X, \mathcal{A}_{1}\right)$ if and only if it is $H A W$ on $\left(X, \mathcal{A}_{2}\right)$.

Proof. The atlases $\mathcal{A}_{1}, \mathcal{A}_{2}$ are compatible if and only if the maximal atlas (with respect to inclusion) $\mathcal{A}_{\max }$ containing $\left(U_{\alpha}, \varphi_{\alpha}\right)$ coincides with the maximal atlas containing $\left(V_{\beta}, \psi_{\beta}\right)$. 
So it is enough to show that $S$ is HAW on $\left(X, \mathcal{A}_{1}\right)$ if and only if it is HAW on $\left(X, \mathcal{A}_{\text {max }}\right)$. For this, apply Proposition 3.3 with $\mathcal{A}=\mathcal{A}_{\max }$ and $\left\{\left(U_{i}, \varphi_{i}\right)\right\}=\mathcal{A}_{1}$.

In light of Corollary 3.4. we will be justified below in omitting the atlas from the terminology and saying that $S \subset X$ is $H A W$ if it is HAW on $(X, \mathcal{A})$ for some atlas of charts $\mathcal{A}$ defining the manifold structure on $X$. It is clear that Proposition 3.1 immediately implies

Proposition 3.5. Let $X$ be a $C^{1}$ manifold. Then:

(a) $H A W$ subsets of $X$ are thick.

(b) The countable intersection of $H A W$ subsets of $X$ is again $H A W$.

(c) The image of a HAW subset of $X$ under a $C^{1}$ diffeomorphism $X \rightarrow X$ is $H A W$.

Now, and for the rest of the paper, let $X$ be as in (1.5) and $F$ as in (1.4). Also define $F^{+} \stackrel{\text { def }}{=}\left\{g_{t}: t \geq 0\right\}$. It turns out that Theorem 1.4 can be reduced to the following statement about one-sided orbits:

Theorem 3.6. $\quad$ (a) The set

$$
\left\{x \in X: F^{+} x \text { is bounded }\right\}
$$

is $H A W$ :

(b) let $Z$ be a compact $\left(F, H^{+}\right)$-transversal submanifold of $X$; then the set

$$
\left\{x \in X: \overline{F^{+} x} \cap Z=\varnothing\right\}
$$

is $H A W$.

Proof of Theorem 1.4 assuming Theorem [3.6. It is clear that a statement analogous to Theorem 3.6 holds for the semigroup $F^{-} \stackrel{\text { def }}{=}\left\{g_{t}: t \leq 0\right\}$ in place of $F^{+}$and with the roles of $H^{+}$and $H^{-}$exchanged: namely, the sets

$$
\left\{x \in X: F^{-} x \text { is bounded }\right\}
$$

and

$$
\left\{x \in X: \overline{F^{-} x} \cap Z=\varnothing\right\},
$$

where $Z$ is a compact $\left(F, H^{-}\right)$-transversal submanifold of $X$, are HAW. The set

$$
\{x \in X: F x \text { is bounded and } \overline{F x} \cap Z=\varnothing\}
$$

is the intersection of sets (3.3)-3.6); hence, in view of Proposition 3.5(b), it is HAW whenever $Z \subset X$ is compact and both $\left(F, H^{+}\right)$- and $\left(F, H^{-}\right)$-transversal. In Theorem 1.4 our set $Z$ is a countable union of manifolds, and hence (replacing if necessary a manifold with a countable union of compact manifolds with boundary) a countable union of compact manifolds with boundary. Thus the set (1.7) is the countable intersection of sets of the form (3.7), and Theorem 1.4 follows by another application of Proposition 3.5(b).

We will finish this section by proving part (a) of the above theorem, which is in fact a rather straightforward variation on some well-known results. In most of the earlier work concerning winning properties of sets of bounded trajectories, the games were actually played on expanding leaves for the $F^{+}$-action on $X$, which in our case can be parametrized as orbits of the expanding horospherical group $H^{+}$. An example is McMullen's strengthening $\mathrm{Mc}$, Theorem 1.3] of a theorem of Dani [D2] on the winning property of the set of directions in hyperbolic manifolds of finite volume with bounded geodesic rays, a special case of which can be restated as follows:

Theorem 3.7. For any $y \in X$, the set

$$
\left\{s \in \mathbb{R}: F^{+} h_{s} y \text { is bounded }\right\}
$$

is absolutely winning (which, for games played on $\mathbb{R}$, is the same as $H A W$ ). 
To reduce Theorem 3.6 (a) to Theorem 3.7, let us fix an atlas of coordinate charts for $X$ as follows. Let $\mathfrak{g} \stackrel{\text { def }}{=} \operatorname{Lie}(G)$, and for any $y \in X$ denote by $\exp _{y}$ the map

$$
\exp _{y}: \mathfrak{g} \rightarrow X, \quad \mathbf{x} \mapsto \exp (\mathbf{x}) y
$$

For any $y \in X$ one can choose a neighborhood $W_{y}$ of $0 \in \mathfrak{g}$ such that $\left.\exp _{y}\right|_{W_{y}}$ is one-to-one. Denote

$$
U_{y} \stackrel{\text { def }}{=} \exp _{y}\left(W_{y}\right) \text { and }\left.\varphi_{y} \stackrel{\text { def }}{=} \exp _{y}^{-1}\right|_{U_{y}} \text {. }
$$

The collection $\left\{\left(U_{y}, \varphi_{y}\right): y \in X\right\}$ is the atlas that we are going to use.

Proof of Theorem 3.6(a). In view of Proposition 3.3 it suffices to show that for any $y \in X$, the set

$$
\varphi_{y}\left(\left\{x \in U_{y}: F^{+} x \text { is bounded }\right\}\right)=\left\{\mathbf{x} \in W_{y}: F^{+} \exp (\mathbf{x}) y \text { is bounded }\right\}
$$

is HAW on $W_{y}$. We know from Theorem 3.7 that the set (3.8) is HAW. Note that conjugation by $g_{t}, t \geq 0$, does not expand elements of $H^{-} F$. Therefore, for any $x \in X$,

$$
F^{+} x \text { is bounded } \Longleftrightarrow F^{+} g x \text { is bounded } \forall g \in H^{-} F \text {. }
$$

The set $U_{y}$ is foliated by connected components of orbits for the action of $H^{-} F$. By composing $\varphi_{y}$ with a suitable diffeomorphism of $W_{y}$, which we are allowed to do by Proposition 3.1(d), we can assume that this foliation is mapped into the foliation of $\mathfrak{g}$ by translates of $\mathfrak{p} \stackrel{\text { def }}{=} \operatorname{Lie}\left(H^{-}\right) \oplus \operatorname{Lie}(F)$. Let us denote by $\mathfrak{h}$ the Lie algebra of $H^{+}$, by $\pi: \mathfrak{g} \rightarrow \mathfrak{h}$ the projection associated with the direct sum decomposition $\mathfrak{g}=\mathfrak{h} \oplus \mathfrak{p}$, and let $W_{y}^{+} \stackrel{\text { def }}{=} \pi\left(W_{y}\right)$.

Fix $0<\beta<1 / 3$. Bob begins with a ball $B_{1} \subset W_{y}$, and Alice consults the strategy she is assumed to have for playing on $W_{y}^{+}$for the chosen value of $\beta$ and taking Bob's first move to be $B_{1}^{\prime} \stackrel{\text { def }}{=} \pi\left(B_{1}\right)$. The strategy specifies an interval (neighborhood of a point) $A_{1}^{\prime} \subset \mathfrak{h}$, and in the game on $W_{y}$ Alice chooses $A_{1} \stackrel{\text { def }}{=} \pi^{-1}\left(A_{1}^{\prime}\right)$, which is a hyperplane neighborhood. Continuing iteratively, suppose Bob has chosen the ball $B_{i} \subset W_{y}$. The ball $B_{i}^{\prime} \stackrel{\text { def }}{=} \pi\left(B_{i}\right)$ is a legal move for Bob playing on $W_{y}^{+}$, since the projection $\pi$ does not affect the radii of balls and since

$$
B_{i} \subset B_{i-1} \backslash A_{i-1} \Longrightarrow B_{i}^{\prime} \subset B_{i-1}^{\prime} \backslash A_{i-1}^{\prime} .
$$

Thus Alice's strategy for playing on $W_{y}^{+}$specifies a move $A_{i}^{\prime} \subset \mathfrak{h}$, and in the new game Alice chooses $A_{i} \stackrel{\text { def }}{=} \pi^{-1}\left(A_{i}^{\prime}\right)$. This defines her strategy for playing on $W_{y}$ and guarantees that $\cap B_{i}^{\prime}$ belongs to the set (3.8). By (3.12), the point $\bigcap B_{i}$ belongs to the set (3.11).

\section{TRAnsversality AND REDUCtion to DisCRETE TIME ACTIONS}

It would seem natural to attempt to prove an analogue of Theorem 3.7 for orbits escaping $Z$, that is, show that for $Z$ as in Theorem 3.6(b) and $y \in X$, the set $\left\{s \in \mathbb{R}: F^{+} h_{s} y \cap Z=\varnothing\right\}$ is HAW. And indeed the above statement is true; however it would not be enough for proving Theorem 3.6 (b). A reason for that is that one can state a version of the equivalence (3.12) for this situation, namely, that

$$
\overline{F^{+} x} \cap Z=\varnothing \Longleftrightarrow \overline{F^{+} \exp (\mathbf{p}) x} \cap Z=\varnothing \quad \forall \mathbf{p} \in \mathfrak{p} \text { with }\|\mathfrak{p}\| \leq \varepsilon,
$$

where $\varepsilon>0$ depends on $x$. However to derive a winning property of the set (3.4) one would need a uniform lower bound on $\varepsilon$, which is not available here. Thus we will need to play on $X$ itself.

Our first step will be a reduction to discrete time actions. The argument here loosely follows [K, §4]. For a parameter $\tau>0$ to be defined later, denote by

$$
F_{\tau}^{+} \stackrel{\text { def }}{=}\left\{g_{n \tau}: n \in \mathbb{Z}_{+}\right\}
$$


the cyclic subsemigroup of $F$ generated by $g_{\tau}$. We will make a reduction showing that we may replace the continuous semigroup $F^{+}$with $F_{\tau}^{+}$. For $Z \subset X$ we define

$$
Z_{\left[t_{1}, t_{2}\right]} \stackrel{\text { def }}{=} \bigcup_{t_{1} \leq t \leq t_{2}} g_{t} Z \text {. }
$$

We have:

Lemma 4.1. Suppose $Z$ is a compact $C^{1}$ submanifold of $X$ and $H$ a connected subgroup of G. Then:

(a) If condition $(F)$ holds, then there exists $\sigma=\sigma(Z)>0$ such that $Z_{[-\sigma, \sigma]}$ is a $C^{1}$ manifold.

(b) If, in addition, condition $(H, F)$ holds, then there exists positive $\tau=\tau(Z) \leq \sigma(Z)$ such that for any $x$ in $Z_{[0, \tau]}, T_{x}(H x)$ is not contained in $T_{x}\left(Z_{[0, \tau]}\right)$.

Note that the conclusion of part (b) of the above lemma coincides with condition $(F)$ with $F$ replaced by $H$ and $Z$ replaced by $Z_{[0, \tau]}$. It will be convenient to introduce more notation and, for a subgroup $H$ of $G$ and a smooth submanifold $Z$ of $X$, say that $Z$ is $H$-transversal at $x \in Z$ if $T_{x}(H x)$ is not contained in $T_{x} Z$, and that that $Z$ is $H$-transversal if it is $H$-transversal at every point of $Z$. In other words, if condition $(F)$ holds with $F$ replaced by $H$. This way, condition $(F)$ says that $Z$ is $F$-transversal, and the conclusion of Lemma 4.1(b) states that $Z_{[0, \tau]}$ is $H$-transversal.

Proof. Let $\operatorname{dim}(Z)=k$. Using a finite covering of $Z$ by appropriate coordinate charts of $X$, one can without loss of generality assume that $Z$ is of the form $\psi(\bar{U})$ for some bounded open $U \subset \mathbb{R}^{k}$, with $\psi$ being a $C^{1}$, nonsingular embedding defined on an open $U^{\prime} \subset \mathbb{R}^{k}$ strictly containing $\bar{U}$. Define $\tilde{\psi}: U^{\prime} \times \mathbb{R} \rightarrow X$ by putting $\tilde{\psi}(u, t)=g_{t}(\psi(u))$. From the $F$-transversality of $Z$ it follows that $\tilde{\psi}$ is nonsingular at $t=0$ and $u \in \bar{U}$. Hence $\tilde{\psi}$ is a nonsingular embedding of $U^{\prime \prime} \times[-\sigma, \sigma]$ into $X$ for some $\sigma>0$ and an open set $U^{\prime \prime}$ strictly containing $\bar{U}$, and (a) is proved.

Clearly the tangent space to $Z_{[-\sigma, \sigma]}$ at $x \in Z$ is equal to $T_{x} Z \oplus T_{x}(F x)$. Therefore condition $(H, F)$ implies that $Z_{[-\sigma, \sigma]}$ is $H$-transversal at any point of $Z$. But the $H$ transversality is clearly an open condition, hence it holds at any point of $Z_{[-\sigma, \sigma]}$ which is close enough to $Z$. By compactness one can choose a positive $\tau \leq \sigma$ such that $Z_{[0, \tau]}$ is $H$-transversal.

Here is another way to express $H$-transversality: fix a Riemannian metric 'dist' on the tangent bundle of $X$, and for a $C^{1}$ submanifold $Z$ of $X$ consider the function $\theta_{H}: Z \rightarrow \mathbb{R}$,

$$
\theta_{H}(x) \stackrel{\text { def }}{=} \sup _{v \in T_{x}(H x),\|v\|=1} \operatorname{dist}\left(v, T_{x} Z\right) .
$$

It is clear that $\theta_{H}(x) \neq 0$ iff $Z$ is $H$-transversal at $x$, and that $\theta_{H}$ is continuous in $x \in Z$. Therefore the following holds:

Lemma 4.2. A compact $C^{1}$ submanifold $Z$ of $X$ is $H$-transversal iff there exist $c=c(Z)>0$ such that $\theta_{H}(x) \geq c$ for all $x \in Z$.

A right-invariant metric on $G$ induces a well-defined Riemannian metric on $X$ and we now change notation slightly, writing 'dist' for the resulting path metric on $X$. Now let $Z$ be as in Theorem 3.6. that is, compact and $\left(H^{+}, F\right)$-transversal, and take positive $\tau \leq \tau(Z)$ as in Lemma 4.1 satisfying in addition:

$$
x, y \in X, 0 \leq t \leq \tau \Rightarrow \operatorname{dist}\left(g_{t} x, g_{t} y\right) \leq 2 \operatorname{dist}(x, y) .
$$

This can be done because $g_{t},|t| \leq \tau$ is bounded and hence there is a uniform bound on the amount by which it distorts the Riemannian metric. Suppose that for some $x \in X$ and $\varepsilon>0$ there exists $t \geq 0$ and $z \in Z$ such that the distance between $g_{t} x$ and $z$ is less than $\varepsilon$. Choose $0 \leq t_{1}<\tau$ such that $t+t_{1}=n \tau$ for some $n \in \mathbb{N}$. It follows from (4.1) that $\operatorname{dist}\left(g_{n \tau} x, g_{t_{1}} z\right)<2 \varepsilon$. This rather elementary argument proves that

$$
\overline{F_{\tau}^{+} x} \cap Z_{[0, \tau]}=\varnothing \Rightarrow \overline{F^{+} x} \cap Z=\varnothing .
$$


Thus to establish Theorem 3.6(b) it suffices to prove the following:

Theorem 4.3. For any compact $H^{+}$-transversal submanifold $Z$ of $X$ and any $\tau>0$ the set

$$
\left\{x \in X: F_{\tau}^{+} x \cap Z=\varnothing\right\}
$$

is $H A W$.

\section{Completion of the Proof: the Percentage Game}

In this final section we prove Theorem 4.3. The argument below originates with an idea of Moshchevitin [Mo], which was developed further in the Ph.D. Thesis of Broderick, and in the papers $\mathrm{B}, \mathrm{BFK}, \mathrm{BFS}$. We follow the streamlined presentation of [BFS, which consists of defining yet another game.

Fix $\beta>0$ and a target set $S \subset \mathbb{R}^{d}$. The hyperplane percentage game is defined as follows: Bob begins as usual by choosing a closed ball $B_{1} \subset \mathbb{R}^{d}$. Then, for each $i \geq 1$, once $B_{i}$ (of radius $r_{i}$ ) is chosen, Alice chooses finitely many affine hyperplanes $L_{i, j}$ and numbers $\varepsilon_{i, j}$, where $j=1, \ldots, N_{i}$, satisfying $0<\varepsilon_{i, j} \leq \beta r_{i}$. Here $N_{i}$ can be any positive integer that Alice chooses. Bob then chooses a ball $B_{i+1} \subset B_{i}$ with radius $r_{i+1} \geq \beta r_{i}$ such that

$$
B_{i+1} \cap L_{i, j}^{\left(\varepsilon_{i, j}\right)}=\varnothing \text { for at least } \frac{N_{i}}{2} \text { values of } j \text {. }
$$

Thus we obtain as before a nested sequence of closed balls $B_{1} \supset B_{2} \supset \cdots$ and declare Alice the winner if and only if (3.1) holds. If Alice has a strategy to win regardless of Bob's play, we say that $S$ is $\beta$-hyperplane percentage winning, or $\beta-H P W$. Note that for large values of $\beta$ it is possible for Alice to leave Bob with no available moves after finitely many turns. However, an elementary argument (see [Mo, Lemma 2] or [BFK, §2]) shows that Bob always has a legal move if $\beta$ is smaller than some constant $\beta_{0}(d)$. In particular $\beta_{0}(1)=1 / 5$. If $S$ is $\beta$-HPW for each $0<\beta<\beta_{0}(d)$, we say that $S$ is hyperplane percentage winning $(H P W)$. It is clear that $\beta$-HPW implies $\beta^{\prime}$-HPW if $\beta \leq \beta^{\prime}$; thus HPW is equivalent to $\beta$-HPW for arbitrary small values of $\beta$.

We remark that the game defined above is actually a special case of the $(\beta, p)$-game defined in $\mathrm{BFS}$, corresponding to the choice $p=1 / 2$. Here $p$ represents the percentage of hyperplanes that Bob is obliged to stay away from.

One sees that in the hyperplane percentage game the rules are more favorable to Alice than in the hyperplane absolute game, and so any HAW set is automatically HPW. Surprisingly, the converse is true, see [BFS, Lemma 2.1]:

Lemma 5.1. For any $\beta \in(0,1 / 3)$ there exists $\beta^{\prime} \in\left(0, \beta_{0}(d)\right)$ such that any set which is $\beta^{\prime}-H P W$ is $\beta-H A W$. In particular the $H P W$ and $H A W$ properties are equivalent.

Proof of Theorem 4.3. Recall that we are given $\tau, \beta>0$ and a compact $H^{+}$-transversal submanifold $Z$ of $X$. Our goal (after using Lemma 5.1 and rewriting $\beta$ for $\beta^{\prime}$ ) is to show that the set (4.2) is $\beta$-HPW. We will assume, without loss of generality, that

$$
\beta<e^{-2 \tau}
$$

Let us say that a map between two metric spaces is $C$-bi-Lipschitz if the ratio of dist $(f(x), f(y))$ and $\operatorname{dist}(x, y)$ is uniformly bounded between $1 / C$ and $C$. Also, for a subset $Y$ in a metric space and $\varepsilon>0$ let us denote by $Y^{(\varepsilon)}$ the $\varepsilon$-neighborhood of $Y$.

The first step is to fix an atlas of coordinate charts for $X$. We will do it as in the proof of Theorem 3.6(a), that is, using charts (3.10) where $\exp _{y}$ is defined by (3.9) and $\left.\exp _{y}\right|_{W_{y}}$ is one-to-one for all $y \in X$. As before, it suffices to show that for any $y \in X$, the set

$$
\varphi_{y}\left(\left\{x \in U_{y}: F_{\tau}^{+} x \cap Z=\varnothing\right\}\right)=\left\{\mathbf{x} \in W_{y}: F_{\tau}^{+} \exp (\mathbf{x}) y \cap Z=\varnothing\right\}
$$

is HAW on $W_{y}$.

The next step is to collect some information about $Z$. Since it is bounded, one can choose $\sigma_{1}<1$ such that the restriction of $\exp _{y}$ to $B_{\mathfrak{g}}\left(0,4 \sigma_{1}\right)$ is 2-bi-Lipschitz (in particular, injective) whenever $B_{X}\left(y, 2 \sigma_{1}\right) \cap Z \neq \varnothing$ (here, as before, we work with a path-metric on $X$ coming from a right-invariant Riemannian metric on $G$ obtained from some inner product 
on $\mathfrak{g}$ ). In light of Corollary 3.4 we can (by replacing the $U_{y}$ with smaller sets, depending on $Z$ ) also assume that $U_{y} \subset B_{X}\left(y, 2 \sigma_{1}\right)$ for any $z \in Z^{\left(4 \sigma_{1}\right)}$. Then, because of compactness and $C^{1}$-smoothness of $Z$, for every $b>0$ there exists $0<\sigma_{2}(b) \leq \sigma_{1}$ such that

$$
\sigma \leq \sigma_{2}(b) \text { and } B_{X}(y, \sigma) \cap Z \neq \varnothing \Rightarrow \text { there exists }
$$

a $\operatorname{dim}(Z)$-dimensional subspace $L$ of $\mathfrak{g}$ such that $\varphi_{y}\left(B_{X}(y, \sigma) \cap Z\right) \subset L^{(b \sigma)}$.

(More precisely, $L$ is the tangent space to $\varphi_{y}(Z)$ at $\varphi_{y}(z)$, where $z$ is some point in the intersection of $B_{X}(y, \sigma)$ and $Z$.) Also, recall that $Z$ is $H^{+}$-transversal, and let $c=c(Z)$ be as in Lemma 4.2 .

Now choose $m$ large enough so that

$$
\beta^{-n}<e^{2 m \tau}, \quad \text { where } n=\left\lfloor\log _{2} m\right\rfloor+1 .
$$

This is possible since the left (resp., right) side of the inequality in (5.3) depends polynomially (resp., exponentially) on $m$.

Pick an arbitrary $y \in X$ and let us suppose that Bob chooses a ball $B_{0} \subset W_{y}$ of radius $r_{0}$. Set

$$
\begin{gathered}
b \stackrel{\text { def }}{=} \frac{c \beta^{n+2} e^{-2 m \tau}}{16}, \\
\sigma \stackrel{\text { def }}{=} \min \left(\frac{1}{4} \sigma_{2}(b), e^{2 m \tau} r_{0}\right),
\end{gathered}
$$

and let

$$
\delta \stackrel{\text { def }}{=} e^{-2 m \tau} \sigma \quad \text { and } \quad \varepsilon \stackrel{\text { def }}{=} b \sigma=\frac{1}{16} c \beta^{n+2} \delta .
$$

We will show that Alice can play the $\beta$-hyperplane percentage game in such a way that the intersection of all the balls belongs to the set

$$
\left\{\mathbf{x} \in W_{y}: F_{\tau}^{+} \exp (\mathbf{x}) y \cap Z^{(\varepsilon)}=\varnothing\right\} .
$$

Note that it follows from (5.5) and (5.6) that $\delta \leq r_{0}$. The game will start with Alice making dummy moves until the first time Bob's ball has radius $r_{1} \leq \delta$ (recall that if the radii $r_{j}$ of the $B_{j}$ do not tend to zero, then Alice wins, see Remark 3.2). Re-indexing if necessary, let us call this ball $B_{1}$ and its radius $r_{1}$; note that we have $r_{1} \geq \beta \delta$.

In order to specify Alice's strategy we will partition her moves into windows. For each $j, k \in \mathbb{N}$, we will say that $k$ lies in the $j$ th window if

$$
\beta^{-n(j-1)} \leq e^{2 k \tau}<\beta^{-j n} .
$$

By (5.3), for every $j \in \mathbb{N}$, there are at most $m$ indices $k$ lying in the $j$ th window, and (5.1) guarantees that every $k \in \mathbb{N}$ lies in some window. We will call the indices $i$ for which

$$
\beta^{n j} r_{1}<r_{i} \leq \beta^{n(j-1)} r_{1}
$$

the $j$ th stage of the game. The first stage begins with Bob's initial ball $B_{1}$, and the rules of the game imply that each stage contains at least $n$ indices. Loosely speaking, Alice will use her moves indexed by numbers in the $j$ th stage, to ensure that the points $\mathbf{x}$ contained in the balls chosen by Bob satisfy $g_{\tau}^{k} \exp (\mathbf{x}) y \notin Z^{(\varepsilon)}$ for any $k$ in the $j$ th window. We now specify Alice's strategy in more detail.

Fix $j$ and suppose that $i=i(j)$ is the first index of stage $j$. For any $k$ belonging to the $j$ th window, denote

$$
A_{j, k} \stackrel{\text { def }}{=}\left\{g_{\tau}^{k} \exp (\mathbf{x}) y: \mathbf{x} \in B_{i}\right\} .
$$

In view of (5.8), the diameter of $A_{j, k}$ is at most

$$
2 e^{2 k \tau} \beta^{n(j-1)} r_{1} \stackrel{(5.7)}{\leq} 2 \beta^{-n} r_{1} \stackrel{(5.3)}{\leq} 2 e^{2 m \tau} r_{1} \stackrel{(5.6)}{\leq} 2 \sigma \stackrel{(5.5)}{\leq} \frac{1}{2} \sigma_{2}(b) .
$$

If $A_{j, k}$ does not intersect $Z^{(\varepsilon)}$ for any $k$ in the $j$ th window, Alice makes all her moves in the $j$ th stage of the game in an arbitrary way (e.g. she could put $N_{i}=0$, that is decide not to specify any hyperplanes on her $i$ th move). Suppose that one of them does; let $x \stackrel{\text { def }}{=} g_{\tau}^{k} y$. 
Then $A_{j, k} \cap B_{X}\left(x, \sigma_{2}(b)\right) \neq \varnothing$. Now let us use $\varphi_{x}$ to map everything to $\mathfrak{g}$. In view of (5.2), there exists $z \in Z$ and a $\operatorname{dim}(Z)$-dimensional subspace $L=T_{\varphi_{x}(z)}\left(\varphi_{x}(Z)\right)$ of $\mathfrak{g}$ such that

$$
\begin{array}{r}
\varphi_{x}\left(A_{j, k} \cap Z^{(\varepsilon)}\right) \subset \varphi_{x}\left(A_{j, k}\right) \cap\left(\varphi_{x} Z\right)^{(2 \varepsilon) \stackrel{\sqrt[5.20]{C}}{\subset}} \varphi_{x}\left(A_{j, k}\right) \cap L^{(2 \varepsilon+2 b \sigma)} \\
\stackrel{\text { [5.4], (5.6) }}{\subset} \varphi_{x}\left(A_{j, k}\right) \cap L^{\left(c \beta^{n+2} e^{-2 m \tau} \sigma / 4\right) .}
\end{array}
$$

In view of Lemma 4.2 and the 2-bi-Lipschitz property of maps $\varphi_{x}$ and $\varphi_{z}$, the intersection of $L^{\left(c \beta^{n+2} e^{-2 m \tau} \sigma / 8\right)}$ with any translate of $\mathfrak{h}$ has length at most $2 \beta^{n+2} e^{-2 m \tau} \sigma$. Consequently, the intersection of

$$
\left\{\mathbf{x} \in B_{i}: g_{\tau}^{k} \exp (\mathbf{x}) y \in Z^{(\varepsilon)}\right\}=\varphi_{y}\left(g_{\tau}^{-k}\left(A_{j, k} \cap Z^{(\varepsilon)}\right)\right)=\operatorname{Ad}_{g_{\tau}^{-k}}\left(\varphi_{x}\left(A_{j, k} \cap Z^{(\varepsilon)}\right)\right)
$$

with any translate of $\mathfrak{h}$ has length at most

$$
2 e^{-2 k \tau} \beta^{n+2} e^{-2 m \tau} \sigma \stackrel{[5.7]}{\leq} 2 \beta^{n(j-1)} \beta^{n+2} \delta \leq 2 \beta^{n(j-1)} \beta^{n+1} r_{1} \leq 2 \beta^{n} r_{i} .
$$

This implies that $\left\{\mathbf{x} \in B_{i}: g_{\tau}^{k} \exp (\mathbf{x}) y \in Z^{(\varepsilon)}\right\}$ is contained in a $\beta^{n} r_{i}$-neighborhood of some hyperplane, and hence the union of sets (5.9) over all indices $k$ contained in the $j$ th window is covered by at most $m \beta^{n} r_{i}$-neighborhoods of hyperplanes. Let us denote these hyperplanes by $L_{i, j}$ and let $\varepsilon_{i, j}=\beta^{n} r_{i}$. These will be Alice's choices in the $i$ th move; the above discussion ensures that they constitute a valid move for Alice. In each of the remaining steps belonging to the $j$ th stage, Alice will choose those neighborhoods which still intersect the ball chosen by Bob. That is, if we write the indices belonging to the $j$ th stage as $i(j), i(j)+1, \ldots, i(j+1)-1$, Alice's choices in stage $j$ will be those hyperplanes $L_{i, j}$ for which $L_{i, j}^{\left(\varepsilon_{i, j}\right)} \cap B_{\ell} \neq \varnothing$, equipped with $\varepsilon_{i, j}=\beta^{\ell} r_{i(j)}$. This choice and (5.8) ensure that all of these moves are valid moves for Alice.

Since the $j$ th stage contains at least $n$ indices, and in every one of his moves Bob must choose a ball intersecting at most $1 / 2$ of the intervals chosen by Alice, we have that out of those neighborhoods, at most $2^{-n} m$ can intersect the first ball $B_{i(j+1)}$ in the first move of the $(j+1)$-th stage of the game. But $2^{-n} m<1$ in view of (5.3). Consequently, for $k$ in the $j$ th window and $\mathbf{x} \in B_{i(j+1)}$, we have that $g_{\tau}^{k} \exp (\mathbf{x}) y$ is not in $Z^{(\varepsilon)}$, and therefore, if $\mathbf{x}_{\infty}$ is the intersection point of all Bob's balls, it follow that $\exp \left(\mathbf{x}_{\infty}\right) y$ belongs to the set (4.2). The theorem is proved.

\section{REFERENCES}

[Ar] C.S. Aravinda, Bounded geodesics and Hausdorff dimension, Math. Proc. Cambridge Philos. Soc. 116 (1994), no. 3, 505-511.

[B] R. Broderick, Incompressibility and fractals, Ph.D. Thesis, Brandeis University, 2011.

[BFK] R. Broderick, L. Fishman and D. Kleinbock, Schmidt's game, fractals, and orbits of toral endomorphisms, Ergodic Theory Dynam. Systems 31 (2011), no. 4, 1095-1107.

[BFS] R. Broderick, L. Fishman and D. Simmons, Badly approximable systems of affine forms and incompressibility on fractals, J. Number Theory 31 (2011), no. 4, 1095-1107.

[BFKRW] R. Broderick, L. Fishman, D. Kleinbock, A. Reich and B. Weiss, The set of badly approximable vectors is strongly $C^{1}$ incompressible, Math. Proc. Cambridge Philos. Soc. 153 (2012), no. 2, 319-339.

[CaSD] J. W. S. Cassels and H. P. F. Swinnerton-Dyer, On the product of three homogeneous linear forms and the indefinite ternary quadratic forms, Philos. Trans. Roy. Soc. London. Ser. A. 248 (1955) 73-96.

[D1] S. G. Dani, Divergent trajectories of flows on homogeneous spaces and Diophantine approximation, J. Reine Angew. Math. 359 (1985), 55-89.

[D2] , Bounded orbits of flows on homogeneous spaces, Comment. Math. Helv. 61 (1986), $636-660$.

[DN] S.G. Dani and A. Nogueira, On orbits of $\mathrm{SL}(2, \mathbb{Z})_{+}$and values of binary quadratic forms on positive integral pairs, J. Number Theory 95 (2002), no. 2, 313-328.

[GP] V. Guillemin and A. Pollack, Differential topology, Prentice Hall (1974).

[J] V. Jarník, Zur metrischen Theorie der diophantischen Approximationen, Prace mat. fiz. 36 (1928), 91-106.

[K] D. Kleinbock, Nondense orbits of flows on homogeneous spaces, Ergodic Theory Dynam. Systems 18 (1998), 373-396. 
[KM] D. Kleinbock and G.A. Margulis, Bounded orbits of nonquasiunipotent flows on homogeneous spaces, Amer. Math. Soc. Translations 171 (1996), 141-172.

[KW1] D. Kleinbock and B. Weiss, Modified Schmidt games and Diophantine approximation with weights, Adv. Math. 223 (2010), no. 4, 1276-1298.

[KW2] _ Modified Schmidt games and a conjecture of Margulis, J. Mod. Dyn., to appear.

[L] C.G. Lekkerkerker, Una questione di approssimazione diofantea e una proprieta caratteristica dei numeri quadratici I, II, Atti Accad. Naz. Lincei. Rend. Cl. Sci. Fiz. Mat. Nat. (8) 21 (1956), 179-185, 257-262.

[Ma1] G.A. Margulis, Dynamical and ergodic properties of subgroup actions on homogeneous spaces with applications to number theory, in: Proceedings of the International Congress of Mathematicians (Kyoto, 1990), pp. 193-215, Math. Soc. Japan, Tokyo, 1991.

[Ma2] G.A. Margulis, Oppenheim Conjecture, in Fields Medallists' lectures, 272-327, World Sci. Ser. 20th Century Math., 5 (1997).

[Mc] C. McMullen, Winning sets, quasiconformal maps and Diophantine approximation, to appear in Geom. Funct. Anal. 20 (2010), 726-740.

[Mo] N.G. Moshchevitin, A note on badly approximable affine forms and winning sets, Moscow Math. J. 11 (2011), 129-137.

[O] A. Oppenheim, Values of quadratic forms. I. Quart. J. Math., Oxford Ser. (2) 4 (1953) 54-59.

[S1] W.M. Schmidt, On badly approximable numbers and certain games, Trans. A.M.S. 123 (1966), $27-50$.

[S2] Badly approximable systems of linear forms, J. Number Theory 1 (1969), 139-154.

[TV] G. Troessaer and A. Valette, On values at integer points of some irrational, binary quadratic forms, in: Essays on geometry and related topics, 597-610, Monogr. Enseign. Math., 38, Enseignement Math., Geneva, 2001.

Department of Mathematics, Brandeis University, Waltham MA 02454-9110, USA

E-mail address: kleinboc@brandeis.edu

Department of Mathematics, Tel Aviv University, Tel Aviv, Israel

E-mail address: barakw@post.tau.ac.il 